\title{
Protonation at the Aqueous Interface of Polymer Nanoparticles with Second Harmonic Generation
}

\author{
Mahamud Subir, Jian Liu, and Kenneth B. Eisenthal* \\ Department of Chemistry, Columbia University, New York, New York 10027
}

Received: May 28, 2008; Revised Manuscript Received: August 7, 2008

\begin{abstract}
The protonation reaction of the carboxyl $(-\mathrm{COOH})$ functional group fixed at the surface of polystyrene carboxylate (PSC) microspheres, with a radius of $457 \pm 14 \mathrm{~nm}$, dispersed in aqueous medium has been studied using the nonlinear optical method of second harmonic generation (SHG). We have demonstrated that the " $\chi^{(3)}$ method," along with the use of the Gouy-Chapman model describing the surface potential, can be used to determine the $\mathrm{p} K_{\mathrm{a}}$ of carboxyl groups at the particle-liquid interface. A surface $\mathrm{p} K_{\mathrm{a}}$ of $5.4 \pm 0.3$ has been determined for the PSC colloidal particles, which corresponds to a decrease in the acidity; that is, $K_{\mathrm{a}}$ is a factor of 4 smaller at the interface than that of long chain carboxyl acids in bulk aqueous solution.
\end{abstract}

\section{Introduction}

Interests in colloidal systems are ever increasing both from the viewpoint of fundamental science and due to their growing applicability in medicine and technology. Because colloidal particles exhibit a large surface area to volume ratio, ${ }^{1,2}$ their chemistry is strongly influenced by their surface properties. They have gained special interest in the issues concerning medical diagnostics and drug delivery systems, ${ }^{3}$ and they are also prominent in the areas of environmental and material science and in chemical manufacturing. ${ }^{2,4}$

Colloidal particles are two phase systems, and the boundary that separates the fluid medium, in which the particle is dispersed, and the particle's inner phase defines the interfacial region. Physical properties such as surface charge density, surface acidity, and surface free energy can and do influence processes such as adsorption and binding of molecules as well as chemical reactions at the surface of the particle. Second harmonic generation (SHG) is a well-established surface selective second order nonlinear optical method that is used in the investigation of various interfaces. In the process of SHG, the incident light, with high peak power, of frequency $\omega$ interacts with the material and induces a second order polarization and thus generates a radiation at twice the frequency of the incident light, that is, $2 \omega$. The nonlinear interaction of the incident light with the chemical species at the interfacial region generates coherent second harmonic radiation that contains information about molecules at the surface and not molecules in the bulk. This selectivity is a direct consequence of the noncentrosymmetry of the interfaces in contrast with bulk media that have centrosymmetric or isotropic symmetry. 5,6 As a result, the method of SHG serves as a selective tool for the study of various chemical interfaces. ${ }^{7,8}$

We have utilized this technique of second harmonic generation to a specific colloidal system in order to study its surface acid-base chemistry. Polystyrene carboxyl (PSC) is a polymer colloid that contains carboxyl, $-\mathrm{COOH}$, functional groups at its surface and thus exhibits acid-base chemistry. In this paper, we report the use of SHG and the " $\chi^{(3)}$ method" to determine

* To whom correspondence should be addressed. E-mail: kbe1@ columbia.edu. the acid-base equilibrium constant for the $-\mathrm{COOH}$ group at the aqueous - particle interface.

\section{Theory}

(1) Acid-Base Chemistry at the Interface. Acid-base reactions are among the most important classes of chemical reactions,

$$
\mathrm{HA}_{(\mathrm{s})}+\mathrm{H}_{2} \mathrm{O}_{(\mathrm{s})} \stackrel{K_{\mathrm{s}}^{\mathrm{s}}}{\rightleftarrows} \mathrm{A}_{(\mathrm{s})}^{-}+\mathrm{H}_{3} \mathrm{O}_{(\mathrm{s})}^{+}
$$

The equilibrium constant, $K_{\mathrm{a}}^{\mathrm{s}}$, for this surface acid-base reaction can be written as

$$
K_{\mathrm{a}}^{\mathrm{s}}=\frac{\left(c_{\mathrm{H}_{3} \mathrm{O}^{+}}^{\mathrm{s}}\right)\left(N_{\mathrm{A}^{-}}^{\mathrm{s}}\right)}{N_{\mathrm{HA}}^{\mathrm{s}}}
$$

where $N_{\mathrm{A}^{-}}^{\mathrm{s}}$ and $N_{\mathrm{HA}}^{\mathrm{s}}$ represent the number densities (molecules per unit surface area) of carboxyl groups, $-\mathrm{COOH}$, and in its ionized form, $-\mathrm{COO}^{-}$, respectively, at the surface of the PSC particle, and $c_{\mathrm{H}_{3} \mathrm{O}^{+}}^{\mathrm{s}}$ is the hydronium ion concentration (moles per unit volume) in the interfacial region. This $\mathrm{H}_{3} \mathrm{O}_{(\mathrm{s})}^{+}$ion concentration near the surface of the particle is related to the bulk concentration of hydronium ions by the Boltzmann factor: ${ }^{1,2}$

$$
c_{\mathrm{H}_{3} \mathrm{O}^{+}}^{\mathrm{s}}=c_{\mathrm{H}_{3} \mathrm{O}^{+}}^{\mathrm{b}} \exp \left[\frac{-e \Phi(0)}{k_{\mathrm{B}} T}\right]
$$

where $\Phi(0)$ is the interfacial potential, $k_{\mathrm{B}}$ is the Boltzmann constant, $T$ is the temperature, $e$ is the magnitude of the electron charge, and $c_{\mathrm{H}_{3} \mathrm{O}^{+}}^{\mathrm{b}}$ is the bulk hydronium ion concentration.

Based on the Gouy-Chapman model for a planar interface, and for a 1:1 bulk electrolyte concentration of $c_{i}\left(\mathrm{~mol} / \mathrm{cm}^{3}\right)$, the surface electric potential, $\Phi(0)$, can be expressed as

$$
\Phi(0)=\frac{2 k_{\mathrm{B}} T}{z_{i} e} \sinh ^{-1}\left[\sigma \sqrt{\frac{\pi}{2 \varepsilon R T c_{i}}}\right]
$$

The symbols $R, \varepsilon, z_{i}$, and $c_{i}$, respectively, are the universal gas constant, the dielectric constant of the medium, the valence of the ion, and the bulk electrolyte concentration of the ionic species $i$. Equation 4 describes the electrostatic potential obtained 
as a solution of the Poisson-Boltzmann equation ${ }^{1}$ for a surface charge density $\sigma$.

It must be emphasized that although the Gouy-Chapman equation is based on a model of charged planar surface, it is applicable to the curved surface of the spherical particle dispersed in electrolyte solution when the product of the inverse of the Debye length, $\kappa$, and the radius of the particle, $r$, is greater than ${ }^{10} 15$, that is, $\kappa r>15$. For the PSC microspheres (diameter $=0.914 \pm 0.027 \mu \mathrm{m}$ ) used in our experiments, we have $\kappa r$ equal to 33 at $1 \mathrm{mM}$ and 336 at $100 \mathrm{mM}$ of total electrolyte concentration.

The carboxyl groups of the PSC particles are fixed at the surface. Thus, the sum of the protonated, $-\mathrm{COOH}$, and the deprotonated, $-\mathrm{COO}^{-}$, forms of the carboxyl functional group is a constant, that is, $N_{\mathrm{A}^{-}}^{\mathrm{s}}+N_{\mathrm{HA}}^{\mathrm{s}}=N_{\text {total }}$. This condition allows us to rewrite eq 2 in terms of the fraction of species that are ionized

$$
K_{\mathrm{a}}^{\mathrm{s}}=\left(c_{\mathrm{H}_{3} \mathrm{O}^{+}}^{\mathrm{s}}\right)\left(\frac{f}{1-f}\right)
$$

in which $f$ is defined as $N_{\mathrm{A}}^{\mathrm{s}}-/ N_{\text {total }}^{\mathrm{s}}$. By combining eqs 3 and 5 , and after taking the negative logarithm of both sides, we obtain an expression for the surface $\mathrm{p} K_{\mathrm{a}}$ in terms of the bulk $\mathrm{pH}$

$$
\mathrm{p} K_{\mathrm{a}}^{\mathrm{s}}=\mathrm{pH}^{\mathrm{b}}+\frac{e \Phi}{2.3 k_{\mathrm{B}} T}+\log \left(\frac{1-f}{f}\right)
$$

In this expression, $\Phi$ is the surface potential, given in eq 4 . Note that the surface charge density, $\sigma$, at a given bulk $\mathrm{pH}$ can be written as $\sigma=\sigma_{\max } f$, where $\sigma_{\max }$ is the maximum surface charge density which can be determined at a high bulk $\mathrm{pH}$ when the maximum numbers of carboxyl groups are ionized.

(2) SHG from the Charged Particle-Aqueous Interface. It is evident from eq 6 that, in order to determine the $\mathrm{p} K_{\mathrm{a}}^{\mathrm{s}}$ and thereby the surface acid-base equilibrium constant at the particle-aqueous interface, one must measure the fraction of molecules that are ionized at the surface of the particle at a given bulk pH. Experimentally, this information is obtainable by detecting the SHG signal that originates from the particleaqueous interface. It must be emphasized that even though a spherical particle, such as the PSC particle discussed in this paper, is centrosymmetric, it can and does generate a second harmonic signal. The second harmonic field, $E_{2 \omega}$, generated at the opposing parts of the particle's surface can add constructively to give a SHG signal if the size of the particle is in the order of the wavelength of the incident light, more precisely related to the coherence length of the process. ${ }^{11}$

The total second harmonic field, $E_{2 \omega}$, originating from a charged interface in contact with water can be expressed as follows:

$$
E_{2 \omega} \propto P_{2 \omega}=\left[\sum_{i} \chi_{i}^{(2)}+\chi_{\mathrm{H}_{2} \mathrm{O}}^{(3)} E_{\mathrm{o}}\right] E_{\omega} E_{\omega}
$$

In this equation, $\chi_{i}^{(2)}$ represents the second order susceptibility of the species $i$ present at the interface, $P_{2 \omega}$ is the second order polarization oscillating at $2 \omega$ induced by the incident fields $E_{\omega}$ and the static field $E_{\mathrm{o}}$, and $\chi^{(3)}$ is the third order susceptibility. The second order susceptibility, $\chi_{i}^{(2)}$, can be written as the product of the number of molecules, $N_{i}^{\mathrm{s}}$, at the surface and the second order polarizability, $\alpha_{i}^{(2)}$, of species $i$, yielding $\chi_{i}^{(2)}=$ $N_{i}^{\S}\left\langle\alpha_{i}^{(2)}\right\rangle_{\mathrm{OR}}$. The bracket, \langle\rangle$_{\mathrm{OR}}$, indicates an orientational average over the interface molecules. ${ }^{12}$

Equation 7 also depicts yet another process by which the second harmonic field is generated from the charged interface. Its source is primarily the interaction of bulk water molecules with the incident light in the presence of the static electric field $E_{\mathrm{o}}$ that exists due to the presence of charged species at the surface. ${ }^{11,13,14}$ This static electric field extends from the interface $(z=0)$ into the bulk solution $(z=\infty)$ polarizing the water molecules, which results in the $\chi^{(3)}$ contribution to the SHG signal. Briefly, both the alignment of the water molecules in the presence of the static electric field and the third order electronic hyperpolarizability, $\alpha_{\mathrm{H}_{2} \mathrm{O}}^{(3)}$, determine the $\chi^{(3)}$ contribution to the SHG signal. The total third order contribution extends from the surface into the bulk solution and is obtained by integration from $z=\infty$ to $z=0$, which is the surface of the particle. This is expressed by

$$
\begin{aligned}
\chi_{\mathrm{H}_{2} \mathrm{O}}^{(3)} E_{\omega} E_{\omega} \int_{0}^{\infty} E_{\mathrm{s}}(z) \mathrm{d} z & =-\chi_{\mathrm{H}_{2} \mathrm{O}}^{(3)} E_{\omega} E_{\omega}[\Phi(\infty)-\Phi(0)] \\
& =\chi_{\mathrm{H}_{2} \mathrm{O}}^{(3)} E_{\omega} E_{\omega} \Phi
\end{aligned}
$$

In this integration we have set the potential to zero at infinity.

At this juncture, we comment on the hyper-Rayleigh (HR) correction that is required for our data analysis. In our experiment, the physical quantity measured is the total intensity of the light at frequency $2 \omega$. Although the measured intensity is mainly due to the SHG from the particle-aqueous interface, there is yet another contribution to the signal at $2 \omega$ from the bulk solution due to HR scattering. The hyper-Rayleigh scattering is an incoherent second order process originating from the breaking of local symmetry due to the fluctuations in the orientation and density of bulk water molecules. ${ }^{15}$ Based on our experimental results, the HR intensity at $2 \omega$ was found to be chiefly from water. The presence of ions in the solution had no effect as demonstrated by the experimental finding that the HR intensity of pure water and electrolyte solution are the same within experimental error. The relative SHG to HR intensities at 1 and $100 \mathrm{mM}$ electrolyte concentration are approximately 2.3 and 1.3 , respectively.

To obtain the SHG signal originating from the particle surface, it is necessary to correct for this HR scattering. Because the hyper-Rayleigh scattering is an incoherent process, and defining $I_{\text {SHG }}$ as the sum of the second harmonic intensities from the individual PSC particles, the total intensity, $I_{\text {total }}$, detected at $2 \omega$ can be expressed as $I_{\text {total }}=I_{\mathrm{SHG}}+I_{\mathrm{HR}}$. The corrected second harmonic field is then given by

$$
E_{\mathrm{SHG}}=\sqrt{I_{\mathrm{SHG}}}=\sqrt{I_{\mathrm{total}}-I_{\mathrm{HR}}}
$$

(3) $\chi^{(3)}$ Method of $\mathbf{p K}_{\mathrm{a}}$ Determination. For the PSC particle-aqueous system, eq 7 becomes

$$
E_{\mathrm{SHG}} \propto\left(\chi_{\mathrm{A}^{-}}^{(2)}+\chi_{\mathrm{HA}}^{(2)}+\chi_{\mathrm{H}_{2} \mathrm{O}}^{(2)}+\chi_{\text {polystyrene }}^{(2)}+\chi_{\mathrm{H}_{2} \mathrm{O}}^{(3)} \Phi\right) E_{\omega} E_{\omega}
$$

where $\chi_{\mathrm{A}}^{(2)}=N_{\mathrm{A}}^{\mathrm{s}}-\left\langle\alpha_{\mathrm{A}}^{(2)}\right\rangle_{\mathrm{OR}}$ and $\chi_{\mathrm{HA}}^{(2)}=N_{\mathrm{HA}}^{\mathrm{s}}\left\langle\alpha_{\mathrm{HA}}^{(2)}\right\rangle_{\mathrm{OR}}$.

The second order susceptibilities, $\chi_{\mathrm{H}_{2} \mathrm{O}}^{(2)}$ and $\chi_{\text {polystyrene, }}^{(2)}$ represent the second order response of water and styrene molecules at the particle surface, respectively. From eq 10, the second harmonic field can be written as

$$
E_{\mathrm{SHG}}=A+B \Phi
$$

where $A$ represents the $\chi^{(2)}$ contributions to $E_{\mathrm{SHG}}$, that is, $A_{\mathrm{HA}}$ $+A_{\mathrm{A}^{-}}+A_{\mathrm{H}_{2} \mathrm{O}}+A_{\text {polystyrene, }}$, and $B$ contains the $\chi^{(3)}$ contribution to $E_{\mathrm{SHG}}$. Since the degree of ionization of the surface carboxyl groups is $\mathrm{pH}$ dependent, the $\mathrm{SHG}$ signal detected as a function of bulk $\mathrm{pH}$ provides information about the fraction of carboxyl groups that are ionized, $N_{\mathrm{A}^{-}} /\left(N_{\mathrm{A}^{-}}+N_{\mathrm{HA}}\right)$. Once this fraction 
is known, eq 6 is applied to determine the $\mathrm{p} K_{\mathrm{a}}^{\mathrm{s}}$ of the carboxyl group at the particle-aqueous interface.

This approach to determine the $\mathrm{p} K_{\mathrm{a}}$ of surface acid-base reactions and, more generally, to investigate the effects of a charged surface on second order spectroscopies is commonly known as the " $\chi{ }^{(3)}$ method" ${ }^{9}$ It is a useful technique to detect and study molecules at a surface that do not make a large $\chi^{(2)}$ contribution to the SHG signal, which is the case with many chemical species. The $\chi^{(3)}$ method has been used successfully in studies of surface potential measurements and $\mathrm{p} K_{\mathrm{a}}$ determinations for various charged interfaces, such as lipid monolayers at the air-water interface and the silica-water interface.9,16,17 It has also been applied to measure the surface charge density of polystyrene sulfate microparticles, emulsions, and charged liposomes and generally colloidal nano-microparticles. ${ }^{18,19}$ Here, we report the first time use of this method to the measurement of acid-base equilibrium constant at a particle interface, namely, for the carboxyl functionalized colloid, polystyrene carboxyl, PSC.

\section{Experimental Section}

Sample Preparation. The polystyrene carboxyl microspheres with diameters of $0.914 \pm 0.027 \mu \mathrm{m}$ were obtained from Polysciences Inc. as $2.61 \%$ solid dispersed in double distilled water. The desired ionic strength and the $\mathrm{pH}$ of the solutions were achieved using $1 \mathrm{mM} \mathrm{HCl}$ (Fisher Scientific), $1 \mathrm{mM} \mathrm{NaCl}$ (EMD Chemical Inc., >99\%), preheated to $600{ }^{\circ} \mathrm{C}$ for more than $6 \mathrm{~h}$, and $1 \mathrm{mM} \mathrm{NaOH}$ (Aldrich, 99.99\%) solutions. All solutions were prepared with doubly distilled water. The final density of the PSC particles was $1.6 \times 10^{8} \mathrm{~cm}^{-3}$ for all the samples. For the $\mathrm{pH}$ dependent experiments, the ionic strength of the solutions was maintained at $1 \mathrm{mM}$.

The $\mathrm{pH}$ value of the samples were measured using a $\mathrm{pH}$ meter (Accumet Basic) $2 \mathrm{~h}$ after sample preparation to ensure complete equilibration of hydronium ions with the carboxyl groups at the particle's surface. Measurements were done both before and after the SHG measurements revealing no changes in the $\mathrm{pH}$ values during the experiment. To verify that there was no $\mathrm{pH}$ dependent swelling or shrinking of the particle, light scattering experiments was carried out using a UV-vis spectrometer (Cary 5000). The intensities of the scattered light from the particle at different $\mathrm{pH}$ values were the same within the experimental error, which suggested that the size of the particles remained the same at different $\mathrm{pH}$ values. All experiments were carried out at room temperature $\left(22^{\circ} \mathrm{C}\right)$.

SHG Setup. The experimental configuration for SHG measurements consisted of a Nd: $\mathrm{YVO}_{4}$ solid state laser (Spectralphysics, Millennia Vs) pumped Ti:sapphire laser (KMLab), which provided $50 \mathrm{fs}$ pulses with an energy of $4 \mathrm{~nJ}$ per pulse at $836 \mathrm{~nm}$ with a repetition rate of $82 \mathrm{MHz}$. The femtosecond laser pulse train was passed through a polarizer and then focused into the sample in a $2 \mathrm{~mm}$ quartz cuvette using a lens with a focal length of $50 \mathrm{~mm}$. A red filter was used between the sample and the lens to block all the stray light at twice the frequency of the laser light. The incident light was S-polarized. The generated SHG signal was collected in the forward direction and sent into a monochromator (CVI). Before the monochromator, a blue filter was used to block the residual fundamental laser light. The detection and data acquisition was made possible using a PC connected to a single photon counter and a photomultiplier tube (PMT).

\section{Results and Discussion}

A prerequisite to the determination of $\mathrm{p} K_{\mathrm{a}}^{\mathrm{s}}$ is the measurement of the maximum surface charge density of the PSC particle.

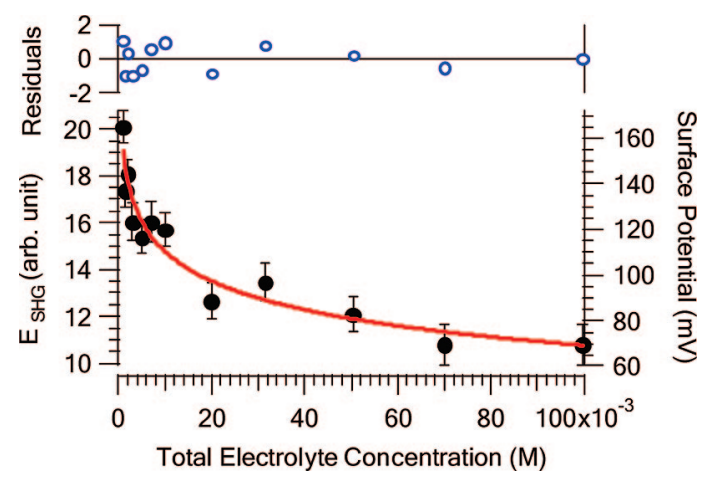

Figure 1. Second harmonic field, $E_{\mathrm{SHG}}$, as a function of bulk electrolyte concentration (M) for the PSC colloids dispersed in water. The black dots are the experimental data, and the red curve is the theoretical fit to eq 12 .

Substitution of eq 4 into eq 11 reveals that the SHG signal originating from the charged PSC particle varies with the bulk electrolyte concentrations (eq 12).

$$
E_{\mathrm{SHG}}=A+B \frac{2 k_{\mathrm{B}} T}{e} \sinh ^{-1}\left[\sigma \sqrt{\frac{\pi}{2 \varepsilon R T c_{i}}}\right]
$$

The maximum ionization of the carboxyl groups is achieved at high $\mathrm{pH}$, yielding the maximum surface charge density $\sigma_{\max }$. Using eq 12 , with the parameters $A, B$, and $\sigma_{\max }$, to fit the $E_{2 \omega}$ dependence on bulk electrolyte concentration allows the determination of the maximum surface charge density.

Figure 1 shows a plot of the second harmonic field as a function of bulk $\mathrm{NaCl}$ concentration. The black dots are the experimental values, and the red curve gives the corresponding fit of the experimental data to eq 12. All solutions at various electrolyte concentration were prepared at bulk $\mathrm{pH}$ of $10.9 \pm$ 0.1 to ensure maximum ionization of the carboxyl groups. According to the fit (see Figure 1), a value of $(3.4 \pm 0.9) \times$ $10^{13}$ charges $/ \mathrm{cm}^{2}$ is obtained for the maximum surface charge density $\sigma_{\max }$. This corresponds to a surface charge density of $(2.9 \pm 0.7) \times 10^{2} \AA^{2}$ per $-\mathrm{COO}^{-}$group at a bulk $\mathrm{pH}$ of 10.9 and a surface coverage of $\sim 9.1 \times 10^{5} \mathrm{COOH}$ species per 0.914 $\mu \mathrm{m}$ PSC particle. The fitting parameters $A$ and $B$ were $6.3 \pm$ 0.2 and $3.8 \pm 0.4$, respectively. Using eq 4 , the surface potential of the PSC particle at an ionic strength of $1 \mathrm{mM}$ was calculated to be $-172 \pm 13 \mathrm{mV}$. For the determination of $\mathrm{p} K_{\mathrm{a}}^{\mathrm{s}}$, the change in the second harmonic signal as a function of bulk $\mathrm{pH}$ was measured. The experimental data are shown in Figure 2. In order to extract the $\mathrm{p} K_{\mathrm{a}}^{\mathrm{s}}$ value, eqs 6 and 12 have been utilized to fit the experimental data. Having determined the maximum surface charge density, we rewrite eq 12 as follows:

$$
E_{\mathrm{SHG}}=A+B \frac{2 k_{\mathrm{B}} T}{e} \sinh ^{-1}\left[\sigma_{\max } f \sqrt{\frac{\pi}{2 \varepsilon R T c_{i}}}\right]
$$

where $f$ represents the fraction of carboxyl groups that are ionized. From eq 6, we obtain

$$
\mathrm{p} K_{\mathrm{a}}^{\varsigma}=\mathrm{pH}^{\mathrm{b}}-\left(\frac{2}{2.3}\right) \sinh ^{-1}\left[f \sigma_{\max } \sqrt{\frac{\pi}{2 \varepsilon R T c_{i}}}\right]+\log \left(\frac{1-f}{f}\right)
$$

To determine the $\mathrm{p} K_{\mathrm{a}}^{\mathrm{s}}$ of the carboxyl group at the PSC particle surface, the $E_{\mathrm{SHG}}$ versus $\mathrm{pH}^{\mathrm{b}}$ data are numerically fitted using eqs 13 and 14 . The $\mathrm{p} K_{\mathrm{a}}^{\mathrm{s}}$ value is found to be $5.4 \pm 0.3$. Figure 2 shows the fit of the experimental results to the theoretical 


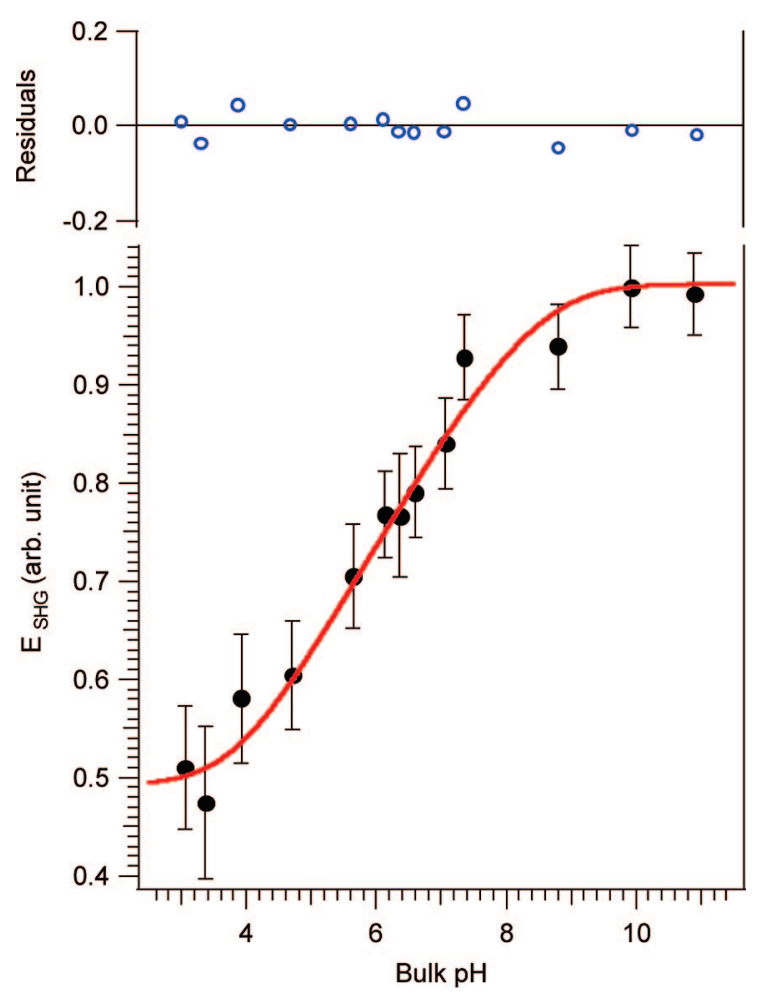

Figure 2. Second harmonic field, $E_{\mathrm{SHG}}$, as a function of bulk $\mathrm{pH}$ for the PSC colloidal particles at a total ionic strength of $1 \mathrm{mM}$. The black dots are the experimental values, and the red curve is the theoretical fit.

expressions relating the measured $E_{\mathrm{SHG}}$ as a function of bulk $\mathrm{pH}$ to the extent of ionization.

The $\mathrm{p} K_{\mathrm{a}}$ values of typical carboxylic acids in aqueous solution at $25{ }^{\circ} \mathrm{C}$ with the number of carbon atoms ranging from 3 to 10 is between 4.8 and $4.9 .{ }^{20}$ Comparing these bulk $\mathrm{p} K_{\mathrm{a}}$ values with the $\mathrm{p} K_{\mathrm{a}}^{\mathrm{s}}$ of the PSC carboxyl group, we find that the acid base equilibrium constant $K_{\mathrm{a}}$ of $-\mathrm{COOH}$ groups at the particle surface is lower than that of the long chain carboxylic acid in aqueous solution by a factor of 4 . This shows that the carboxyl groups are less acidic at the particle-liquid interface. This is a consequence of greater surface free energy due to the increase in repulsive interactions between the charged species as the neutral carboxyl groups ionize. Hence, the acid-base equilibrium at the particle surface favors the neutral form, that is, the protonated form of the carboxyl group, resulting in a higher $\mathrm{p} K_{\mathrm{a}}$ at the interface relative to the $\mathrm{p} K_{\mathrm{a}}$ of similar chemical species in the bulk solution. The free energy, $\Delta G^{\mathrm{s}}$, for the acid-base reaction at the particle surface is $30 \pm 2 \mathrm{~kJ} / \mathrm{mol}$.

\section{Conclusion}

We have demonstrated that the nonlinear optical technique of second harmonic generation, particularly the " $\chi^{(3)}$ method", can be applied to study acid-base chemistry at the particle-liquid interface. The acid-base property, $\mathrm{p} K_{\mathrm{a}}^{\mathrm{s}}$, of the $-\mathrm{COOH}$ moiety of the polystyrene carboxylate microsphere has been determined using this method, and it has been found that the carboxyl group fixed at the particle surface is more basic compared to similar carboxylic acids in bulk aqueous medium. This shift in the acid-base equilibrium constant by a factor of 4 is attributed to higher surface free energy of the charged particle-liquid interface. This serves as direct evidence that the surface properties of colloidal systems strongly influence the chemistry that occurs at the colloidal particle surface.

Acknowledgment. We gratefully acknowledge support from the National Science Foundaion, the Chemical Sciences, Geosciences and Bioscience Division, Office of Basic Energy Sciences, Office of Science of the U.S. Department of Energy, and DTRA(W911NF-07-1-0116).

\section{References and Notes}

(1) Heimenz, P. C. Principle of Colloid and Surface Chemistry, 2nd ed.; Marcel Dekker, Inc.: New York, 1986.

(2) Butt, H.-J.; Graf, K.; Kappl, M. Physics and Chemistry of Interfaces, 2nd ed.; Wiley-VCH Verlag GmbH \& Co. KGaA: Weinheim, 2006.

(3) Singh, M.; Briones, M.; Ott, G.; O'Hagan, D. Proc. Natl. Acad. Sci. U.S.A. 2000, 97, 811.

(4) Fitch, R. M. Polymer Colloids: A Comprehensive Introduction; Academic Press, Inc.: New York, 1997.

(5) Bloembergen, N. Nonlinear Optics; Wiley: New York, 1965.

(6) Shen, Y. R. The Principles of Nonlinear Optics; John Wiley \& Sons, Inc.: New York, 2003.

(7) Eisenthal, K. B. Annu. Rev. Phys. Chem. 1992, 43, 627.

(8) Eisenthal, K. B. Chem. Rev. 2006, 106, 1462.

(9) Zhao, X.; Ong, S.; Wang, H.; Eisenthal, K. B. Chem. Phys. Lett. 1993, 214, 203.

(10) White, L. R. J. Chem. Soc., Faraday Trans. 2 1977, 73, 577.

(11) Ong, S.; Zhao, X.; Eisenthal, K. B. Chem. Phys. Lett. 1992, 191, 327.

(12) Eisenthal, K. B. Chem. Rev. 1996, 96, 1343.

(13) Xiaolin, Z.; Ong, S.; Eisenthal, K. B. Chem. Phys. Lett. 1993, 202, 513.

(14) Levine, B. F.; Bethea, C. G. Appl. Phys. Lett. 1975, 26, 375.

(15) Clays, K.; Persoons, A. Phys. Rev. Lett. 1991, 66, 2980.

(16) Wang, H. F.; Zhao, X. L.; Eisenthal, K. B. J. Phys. Chem. B 2000, 104, 8855 .

(17) Konek, C. T.; Musorrafiti, M. J.; Al-Abadleh, H. a.; Bertin, P. A.; Nguyen, S. T.; Geiger, F. M. J. Am. Chem. Soc. 2004, 126, 11754.

(18) Yan, E. C. Y.; Liu, Y.; Eisenthal, K. B. J. Phys. Chem. B 1998, $102,6331$.

(19) Liu, Y.; Yan, C. Y.; Zhao, X. L.; Eisenthal, K. B. Langmuir 2001, 17, 2063.

(20) Christensen, J. J.; Hansen, L. D.; Izatt, R. M. Handbook of Proton Ionization Heats and Related Thermodynamic Quantities; John Wiley \& Sons: New York, 1976.

JP8047168 